Estudios de la Paz y el Conflicto

Revista Latinoamericana

IUDPAS-UNAH

Número Especial 1, pp. 27-42

ISSN-e: 2707-8922 / ISSN: 2707-8914

DOI: $10.5377 /$ rlpc.v0i 0.9501

Anual 2019

\title{
ENFOQUE DE DERECHOS HUMANOS: UNA HERRAMIENTA PARA LA CONSTRUCCIÓN DE PAZ Y UN MEDIO PARA EL DESARROLLO DE OTROS ENFOQUES
}

\author{
HUMAN RIGHTS-BASED APPROACH: A TOOL FOR PEACE BUILDING AND A \\ MEANS FOR THE DEVELOPMENT OF OTHER APPROACHES
}

\author{
Natali Niño Patiño \\ Universidad Libre de Colombia \\ natali.ninop@unilibre.edu.co \\ Laura Milena Aldana González \\ Universidad Libre de Colombia \\ lauram-aldanag@unilibre.edu.co \\ María Camila Zuluaga \\ Universidad Libre de Colombia \\ mariac-zuluagag@unilibre.edu.co
}

Cómo citar / citation

Niño, N., Aldana, LM. y Zuluaga, MC. (2019). "Una herramienta para la construcción de paz y un medio para el desarrollo de otros enfoques", Estudios de la Paz y el Conflicto, Revista Latinoamericana, Número Especial 1, 27-42. DOI: 10.5377/rlpc.v0i0.9501

\section{Resumen}

El presente documento tiene como propósito principal determinar qué se puede comprender por el enfoque de derechos humanos, a su vez mostrar cómo en la materialización de una política pública como lo fue la construcción del Acuerdo Final de Paz, el enfoque de derechos humanos permitió la apertura a la construcción de otros enfoques, conocidos como enfoques diferenciales, los cuales se instalan en el discurso jurídico con el fin de ser obligatorios tanto en la construcción de política pública como en la toma de decisión judicial, lo cual permitirá el desarrollo del principio a la paz.

\section{Palabras claves}

Enfoque de derechos humanos; Política Pública; Decisiones judiciales; Enfoques diferenciales. 


\begin{abstract}
The main purpose of this document is to determine what can be understood as the human rights approach, and at the same time to show how in the materialization of a public policy, such as the construction of the Acuerdo Final de Paz, the human rights approach allowed the opening to the construction of other approaches, known as differential approaches, which are installed in the legal discourse in order to be obligatory both in the construction of public policy and in judicial decision making, which will allow the development of the principle of peace.
\end{abstract}

\title{
Palabras clave
}

Human rights-based approach; Public politics; Judicial decisions; Differential approaches.

\section{INTRODUCCIÓN}

Colombia y la mayoría de los estados en América Latina reconocen los derechos humanos como aquellos limites inquebrantables de todos los ciudadanos ${ }^{1}$, qué desde la definición de Ferrajoli (2012) estos (los derechos) deben estar adscritos universalmente y deben evitar ser afectados, además son considerados como preceptos ético-morales ${ }^{2}$ que guían todas las circunstancias y legitiman su existencia. No obstante, es cuestionable el verdadero respeto de los estados y la materialización de los derechos humanos, si tenemos en cuenta los contextos actuales, la situación social, política y económica del mundo o si pensamos en países más cercanos como Chile, Ecuador, Venezuela, Argentina, Bolivia, Colombia, entre otros, que se encuentran en crisis por diferentes causas, en donde la protesta y manifestaciones se convierte en un vehículo para defender, -por parte de la ciudadanía- los derechos como la educación, la salud, la vivienda, el trabajo, y demás, creando así marcos de conflictos entre la ciudadanía y el Estado. Así las cosas, podemos inferir, a partir de lo anterior, que los gobiernos están fallando en garantizar y hacer efectivos los derechos humanos, y con ello las constituciones, los convenios y tratados internacionales que ratifica y se compromete a cumplir, en el marco de la defensa y protección de los derechos de todas las personas.

Los derechos humanos son producto de importantes sucesos históricos como la Revolución Inglesa, de la que surgió el Bill of Rights en 1688; de la Revolución Norteamericana, con la Declaración de Derechos del Estado de Virginia en 1776; la Revolución Francesa de 1789, con la Declaración de los Derechos del Hombre y el Ciudadano; la Constitución de Querétaro en 1917; y, además de la conciencia que deja represión y violencia de los sucesos del siglo XX en América Latina, además de innumerables luchas sociales que contribuyeron al reconocimiento de derechos que aún se encuentran

\footnotetext{
${ }^{1}$ A la pregunta sobre qué son los Derechos Humanos, Ferrajoli responde que son aquellos que "los identifica con los derechos que están adscritos universalmente a todos en cuanto personas, o en cuanto ciudadanos o personas con capacidad de obrar, y que son por tanto indisponibles e inalienables" (Ferrajoli, 2012). La Corte Interamericana de Derechos Humanos, en adelante, Corte IDH, ha señalado que se hace necesario que los Estados protejan, cumplan y hagan efectivo los derechos humanos como límite al poder que le es otorgado.

${ }^{2}$ Los derechos humanos son considerados como valores universales que se materializan en las constituciones y en el corpus iuris internacional. Detrás de esta postura existe una discusión sobre la forma en cómo se materializan y se cumplen, para ello, recomendamos la discusión entre el ius positivismo y el ius naturalismo. Estudios de la Paz y el Conflicto, Revista Latinoamericana, IUDPAS-UNAH, Número Especial 1, 2019, pp. 27-42. DOI: 10.5377/rlpc.v0i0.9501
} 
vigentes dentro de nuestras legislaciones y que fueron posteriormente consagrados dentro de convenios y tratados internacionales, estableciendo un conjunto de garantías para todos los seres humanos sin distinción alguna, que orientan las acciones u omisiones de los estados, con el fin de proteger la dignidad humana y el pleno desarrollo de la vida.

Específicamente en el caso colombiano, en el año 2016 se firmó el Acuerdo Final para la terminación del conflicto y la construcción de una paz estable y duradera (en adelante, Acuerdo Final de Paz), en el que se buscaba salir del conflicto armado generado entre el Estado Colombiano y la guerrilla más antigua de América Latina, las Fuerzas Armadas Revolucionarias de Colombia (FARC- EP). La construcción del documento implicó un esfuerzo por parte de la comunidad, las víctimas, las mujeres, los campesinos, el Estado, la guerrilla, entre otros actores, quienes de la mano con la comunidad internacional consolidaron el fin conflicto y generaron un documento que respondiera a las exigencias de la comunidad, implementando nuevas instituciones, las cuales no se reducían a puntos concretos del desarme de la guerrilla, sino el documento es una garantía para superar la violencia estructural (Galtung, 1998) que existen en Colombia, de forma especial en el campo.

Por consiguiente, los enfoques se constituyen como un método ${ }^{3}$ o un paso a paso, por el cual se busca desarrollar política pública estatal y proferir decisiones judiciales, teniendo en cuenta los derechos humanos y los riesgos particulares de cierta parte de la población, es decir, que cada vez que se busque desarrollar una política pública deba acoplarse con los estándares de derechos humanos.

Por lo tanto, el presente artículo tiene como propósito principal responder ¿Qué es el enfoque de derechos humanos en la construcción del Acuerdo Final de Paz como una política pública Estatal? Para responder a la anterior pregunta, el artículo se desarrollará en tres partes, en la primera se explicará la definición y contexto de los derechos humanos en la actualidad, en la segunda, se realizará un análisis de cómo se ha desarrollado el enfoque de derechos humanos y por último, se revisará cuál es el desarrollo del enfoque de derechos humanos en el Acuerdo Final de Paz y cómo este permitió la construcción de otros enfoques diferenciales en Colombia.

\section{METODOLOGÍA}

El método utilizado para el desarrollo del trabajo es el análisis de texto, revisando textos tanto de fuente primaria como secundaría, incluyendo textos de diferentes disciplinas como lo son, la sociología y la teoría del derecho, lo que habilitará la justificación de la construcción del concepto, esto es la conceptualización del enfoque de derechos humanos, para así establecer qué debemos entender por el enfoque dentro de la construcción de una política pública del Estado para la paz en Colombia y lo que debemos entender por los enfoques diferenciales que emergen en el Acuerdo Final de Paz.

El presente artículo se encuentra en el marco de la investigación denominada "Perspectivas pedagógicas: Herramientas para la construcción de ambientes de paz en Colombia", la cual comenzó a desarrollarse desde el año 2017, en el que se introduce como

\footnotetext{
${ }^{3}$ En el texto la profesora Niño (2019) realiza una distinción entre enfoque y perspectiva. En el que considera que el enfoque es el método de aplicación en la política pública y la perspectiva son las teorías que se desarrollan para justificar la inclusión de cada uno de los enfoques.

Estudios de la Paz y el Conflicto, Revista Latinoamericana, IUDPAS-UNAH, Número Especial 1, 2019, pp. 27-42. DOI: $10.5377 /$ rlpc.v0i0.9501
} 
metodología principal, realizar un análisis del discurso de, por un lado, las fuentes primarias ${ }^{4}$, como la lectura crítica del Acuerdo Final de Paz, y la participación en la implementación del Acuerdo $^{5}$, desde las tribunas del Congreso de la República de Colombia, en las discusiones del procedimiento legislativo especial para la paz y por otro lado, las fuentes secundarias ${ }^{6}$, desde la teoría sociológica como lo es el concepto de violencia de Galtung, y las implicaciones filosóficos que tiene el acuerdo para el derecho, entre otras.

Así las cosas, al realizar un análisis del desarrollo de la implementación del Acuerdo Final de Paz, y esto al mismo tiempo contrastarlo con el concepto de violencia diseñado por Galtung (1998), y el desarrollo ius filósofico de los derechos humanos, desde la teoría de Ferrajoli, hasta el análisis jurisprudencial de la Corte Interamericana de Derechos Humanos, (en adelante, Corte IDH) y de la Corte Constitucional Colombiana (en adelante Corte Constitucional), nos permite comprender los mecanismos instalados en el Acuerdo y las herramientas jurídicas y políticas, para conceptualizarlos desde una perspectiva crítica, de qué debe entenderse por el enfoque de derechos humanos, cuál ha sido el impacto en la implementación del acuerdo y a su vez, de cómo debe utilizarse en el desarrollo de la política del Estado y la toma de decisión judicial.

El objetivo general del artículo es conceptualizar el enfoque de los derechos humanos en la construcción del Acuerdo Final de Paz como una política pública Estatal, para lograr lo anterior, el texto se dividirá en tres partes, partes que hacen referencia a los objetivos específicos, así:

- Determinar el fundamento teórico y jurídico del concepto de los derechos humanos en la teoría de Ferrajoli (2012) y en la jurisprudencia de la Corte IDH.

- Analizar el desarrollo conceptual del enfoque de derechos humanos.

- Determinar el impacto que generó el enfoque de los derechos humanos en la materialización del Acuerdo final de paz, entendiendo el acuerdo como una política pública estatal.

\section{LOS DERECHOS HUMANOS: LÍMITES AL EJERCICIO DEL PODER}

El presente acápite tiene como objetivo principal establecer cuáles son los fundamentos teóricos y jurídicos de los derechos humanos, esto nos permitirá entender después cómo se consolida el enfoque de derechos humanos. Este objetivo se cumplirá a la luz de la teoría de los derechos fundamentales de Luigi Ferrajoli y de jurisprudencia constitucional y convencional entorno al concepto.

\footnotetext{
${ }^{4}$ Se entiende las fuentes primarias aquellos documentos que desencadenaron toda una suerte de sucesos en Colombia.

${ }^{5}$ En el marco de la investigación, el Observatorio de Paz, realizó un seguimiento activo desde las tribunas del Congreso de la República, en el marco de la implementación del procedimiento legislativo especial para la paz, estableciendo como mirada un análisis crítico del discurso entorno a la implementación y a los mecanismos desarrollados en el procedimiento.

${ }^{6}$ En el desarrollo del presente artículo -si bien es cierto- no se encuentran de forma directa las citas y fuentes utilizadas para la realización del diagnóstico, sí es importante enmarcarlo dentro del cuadro de investigación, en donde se han realizado otros aportes, como la edición de libros y la publicación de artículos, en el que se ha revisado el esfuerzo de encontrar las herramientas que se construyeron desde la implementación del acuerdo para generar paz en los territorios y en los diferentes espacios de la sociedad.

Estudios de la Paz y el Conflicto, Revista Latinoamericana, IUDPAS-UNAH, Número Especial 1, 2019, pp. 27-42. DOI: $10.5377 /$ rlpc.v0i0.9501
} 
Luigi Ferrajoli es uno de los teóricos más importantes en la teoría contemporánea del derecho, su teoría la ha denominado como el complemento del positivismo jurídico (Ferrajoli, 2012), en la medida en que a partir de herramientas internas del derecho, se puede revisar lo que él denomina como la legitimidad del derecho, esto es ver cuándo estamos ante normas que son formalmente validas, pero materialmente invalidas, por consiguiente, las normas dentro de un ordenamiento jurídico dependen de no vulnerar derechos fundamentales que se encuentran dentro de la constitución, denominando su teoría como positivismo crítico o positivismo garantista.

Entonces, para Farrojoli los derechos fundamentales son considerados como derechos universales, "es decir conferidos a todos por normas jurídicas y derechos subjetivos" (2016: 34). En otras palabras, derechos contemplados en ordenamientos jurídicos o en declaraciones o convenciones internacionales, que a su vez requieren de mecanismos que permitan su desarrollo, bien sea, por un lado, de prohibición, o, por otro lado, de prestación. Ferrajoli utiliza la categoría de derechos fundamentales porque considera que:

"nos referimos, en este sentido, al fundamento de la definición teórica del concepto de derechos fundamentales. Por lo que a mí respecta, como ha recordado Camanducci, me refiero con derechos fundamentales todos esos derechos que son adscribibles universalmente a todos en cuanto a personas, o en cuanto a ciudadano o en cuanto capaces de actuar y que son, por tanto, indisponibles e inalienables." (2016: 36)

Hay una diferencia conceptual entre los derechos humanos y los derechos fundamentales que es útil al momento de comprender la discusión teórica entre lo que Ferrajoli (2012), denomina constitucionalismos garantistas y constitucionalismo argumentativo ${ }^{7}$. Los derechos humanos son todos aquellos derechos que se consideran esenciales al ser humano, esta concepción propiamente viene de la idea esencialista de la naturaleza humana y los derechos fundamentales son aquellos reconocidos en las constituciones, lo cual hace que tengan titularidad y, por lo tanto, puedan ser ejercidos por medio del derecho subjetivo.

Esta conceptualización se hace confusa -en el caso colombiano- al momento de comprender los documentos jurídicos concretos, porque hay un catálogo de derechos fundamentales que se reducen a los derechos individuales y políticos, aunado que en Colombia hacen parte de la Constitución varios instrumentos internacionales, los cuales hablan de derechos humanos, por ende, para fines de este artículo, se entenderá por derechos humanos lo mismo que por derechos fundamentales, esto es, aquellos que son reconocidos en la Constitución Política de Colombia, y que por medio de tratados y convenios hacen parte integral de la misma, en conclusión, se vuelven obligatorios y de exigencia para el Estado. Es importante aclarar que los derechos humanos no son únicamente un catalogo básico de derechos, sino son todos aquellos principios o mejor en palabras de Alexy, son aquellas normas de textura abierta que integran el ordenamiento jurídico (Alexy, 2008).

Los derechos humanos, según la Organización de las Naciones Unidas (en adelante ONU), se "conocen como derechos inherentes a todos los seres humanos, sin distinción alguna de

\footnotetext{
${ }^{7}$ Ferrajoli (2012) señala que para avanzar en la discusión sobre "ius naturalismo" y "ius positivismo", debería establecerse nuevos conceptos que permitan comprender la referencia contemporánea de los teóricos, por un lado, señala el constitucionalismo que el propone como constitucionalismo garantista y el constitucionalismo que defiende Atienza (2019), entre otros, como constitucionalismo argumentativo, en el sentido, que las teorías de la argumentación entran a completar el sistema del derecho, cuando nos encontramos ante lo que Hart (2004), denomina como casos fáciles y casos difíciles y Atienza complementa con los casos intermedios y casos trágicos (Atienza, 2017). Estudios de la Paz y el Conflicto, Revista Latinoamericana, IUDPAS-UNAH, Número Especial 1, 2019, pp. 27-42. DOI: $10.5377 /$ rlpc.v0i0.9501
} 
raza, sexo, nacionalidad, origen étnico, lengua, religión o cualquier otra condición" (ONU, 1948), los cuales nadie puede vulnerar, en especial el Estado que tiene como fundamento la defensa y protección de estos preceptos básicos que permiten crear las condiciones indispensables para que las personas puedan vivir dignamente.

Las características de los derechos humanos son primero, que estos deben ser universales. Esta característica hace referencia a su reconocimiento, aceptación y aplicación por todos los estados, aun así, en la doctrina se ha cuestionado este planteamiento desde el relativismo como concepción ontológica del mundo- en el que se plantea que los valores no son universales; los valores surgen desde las culturas, sin embargo, el enfoque de los derechos humanos, reconoce el multiculturalismo, lo que quiere decir que el desarrollo e interpretación es diferente dentro de las distintas culturas y los derechos variarían según los contextos culturales. Ejemplo de ello, es el concepto de vivienda digna para las personas que viven en la ciudad y las comunidades indígenas.

La segunda característica es que son indivisibles, esto es que ningún derecho puede prescindirse o considerarse de mayor importancia que otro. Esta característica también se ha cuestionado debido a que existen unos derechos estructurales como la vida o libertad y sin ellos, sería imposible garantizar otros como los derechos económicos y políticos, en la práctica usualmente se priorizan algunos derechos.

Otra característica es que son inalienables e irrenunciables; esta hace referencia a la imposibilidad de ser enajenados y a renunciar a ellos, aunque sea por la propia voluntad de las personas.

En Colombia las disposiciones contenidas en convenios y tratados internacionales que versan sobre derechos humanos se adoptan a través de una figura llamada Bloque de Constitucionalidad, es decir, según la Sentencia de la Corte Constitucional de Colombia C067, de 4 de febrero de 2003, "son normas y principios que, sin aparecer formalmente en el articulado del texto constitucional, son utilizados como parámetros del control de constitucionalidad de las leyes". Esta figura se encuentra en el artículo 93 de la Constitución Política de Colombia de 1991, la cual le otorga un carácter vinculante a estos instrumentos, lo que implica que, por haber sido ratificados por el Estado, son de obligatorio cumplimiento y pasan a ser parámetros de interpretación.

Es importante recordar que no todos los tratados ratificados por Colombia hacen parte del Bloque de Constitucionalidad, sino los que versan sobre Derechos Humanos y, "que no pueden ser limitados en estados de excepción" (art. 93 de la Constitución Política de Colombia de 1991), aunque se fueron agregando otros como tratados limítrofes, convenios OIT y más. Los mecanismos por los cuales se hacen efectivas estas disposiciones en el ordenamiento jurídico interno son el control de constitucionalidad que realiza la Corte Constitucional Colombiana, y los jueces cuando se encuentran con normas que violan la Constitución Política, y el control de convencionalidad, que está en cabeza de la Corte IDH, cuando Estados violan preceptos establecidos en convenciones que se han comprometido a cumplir.

En resumen, estas normas que tienen rango constitucional integrando el bloque superior, cumplen las siguientes finalidades según Bobbio (1957: 887):

1. "Regla de interpretación respecto a las dudas que puedan suscitarse al momento de su aplicación.

2. La de integrar la normatividad cuando no exista norma directamente aplicable al caso.

3. La de orientar las funciones del operador jurídico.

Estudios de la Paz y el Conflicto, Revista Latinoamericana, IUDPAS-UNAH, Número Especial 1, 2019, pp. 27-42. DOI: 10.5377/rlpc.v0i0.9501 
4. La de limitar la validez de las regulaciones subordinadas".

Teniendo en cuenta lo anterior, se establece que los tratados y convenios de derechos humanos son fuente de derecho y cuentan con jerarquía constitucional, es decir, que los jueces y todos los funcionarios del Estado deben atenerse a sus prescripciones ajustando leyes, actos administrativos y decisiones judiciales.

Los derechos humanos, además de ser garantías de todos los individuos, son una importante forma para limitar las arbitrariedades del poder de los Estados, cuando estos, a través de acciones u omisiones precarizan su cumplimiento. En el Estado colombiano recae la obligación de materializar los derechos humanos, sin embargo, existe subsidiariamente la acción de los tribunales regionales que en caso de agotar instancias internas que no resuelven una situación de violación de derechos, pueden condenar a los Estados, para que estos modifiquen lo que permitió la trasgresión, y adopten las medidas necesarias para resarcir a las víctimas.

Un ejemplo de ello se presenta en la Sentencia de la Corte Interamericana de Derechos Humanos del 15 septiembre de 2005 (caso de La Masacre de Mapiripan Vs. Colombia), que fue aceptado por la Comisión Interamericana de Derechos Humanos, y remitido a la Corte IDH en la cual se determinó la responsabilidad del Estado Colombiano por haber omitido la investigación correspondiente de la masacre cometida por parte de grupos paramilitares en el territorio de Mapiripán.

En este caso, se demostró una vinculación de las Fuerzas Armadas con grupos paramilitares, cuando estos últimos aterrizaron en San José de Guaviare con aquiescencia de militares, facilitando su traslado a Mapiripán, allí el grupo paramilitar torturó y asesinó a habitantes de la población. La fuerza pública llegó al lugar siete días después de la masacre, cuando ya se había destruido la evidencia física y elementos materiales probatorios. No hubo posteriormente sanciones ni investigación alguna por parte del Estado.

La CIDH determinó que se habían violado varios artículos de la Convención Interamericana de Derechos Humanos, la Convención Interamericana para Prevenir y Sancionar la Tortura, los Convenios de Ginebra de 1949 y el protocolo I adicional, entre otros instrumentos internacionales a los cuales Colombia se había comprometido a cumplir. Además, estableció medidas restitutivas, pecuniarias, de satisfacción y no repetición que el Estado de Colombia debía adoptar por haber quebrantado el cumplimiento de los instrumentos mencionados. También en esta misma sentencia (caso de La Masacre de Mapiripan Vs. Colombia) se reiteró que "la Convención Interamericana de Derechos Humanos en el marco del Derecho Internacional de los Derechos humanos, se inspiran en valores comunes superiores (centrados en la protección del ser humano), están dotados de mecanismos específicos de supervisión, se aplican de conformidad con la noción de garantía colectiva y consagran obligaciones de carácter objetivo" (CIDH, 2005: parágrafo: 104).

Por otra parte, en la Sentencia de la Corte Constitucional de Colombia C-579/13, de 28 de agosto de 2013, la Corte Constitucional relata que el cumplimiento de los derechos humanos es una de las principales funciones del Estado Social de Derecho, y la obligación de cumplimiento de estos comprende: prevenir su vulneración, elaborar mecanismos adecuados para la protección de los derechos cuando exista una vulneración o amenaza, reparar las violaciones y "esclarecer los hechos, investigar, juzgar y sancionar las violaciones a los derechos humanos y al Derecho Internacional Humanitario" (Corte Constitucional, Sentencia C-579/13).

Estudios de la Paz y el Conflicto, Revista Latinoamericana, IUDPAS-UNAH, Número Especial 1, 2019, pp. 27-42. DOI: 10.5377/rlpc.v0i0.9501 
Podemos entender, según establecen las cortes en sus providencias, que las acciones de los Estados están previamente limitadas por los derechos humanos, y es sancionada toda arbitrariedad que fragmente el debido desarrollo de estos. Es por esto que su aplicación y materialización requieren de un mecanismo para que los derechos dejen de ser proyectos sustanciales y sean implementados correctamente para que sus titulares eviten ser vulnerados por su falta de cumplimiento.

Finalmente, para la garantía y satisfacción idónea de los derechos humanos, se deben reconocer las diferencias de cada uno de sus titulares, pues deben implementarse en distintas medidas para que no sean vulnerados. Este reconocimiento de las diferencias que nos hace humanos, es la que busca atender el enfoque de derechos humanos, para que los derechos sean instrumentos vivos que respondan a las características de todos y todas de manera precisa, pues "la pluralidad es la condición de la acción humana debido a que todos somos lo mismo, es decir, humanos, y por tanto nadie es igual a cualquier otro que haya vivido, viva o vivirá" (Arendt, 2012: 36).

\section{DESARROLLO JURÍDICO DEL ENFOQUE DE DERECHOS HUMANOS}

El presente acápite tiene como propósito principal conceptualizar el enfoque de derechos humanos y cómo este se ha desarrollado en el marco jurídico. Así las cosas, los defensores de derechos humanos, académicos y miembros de la organizaciones internacionales en especial, la ONU, buscan implementar herramientas y mecanismos útiles para lograr un cambio social revindicando los derechos de todos los individuos, por lo tanto, surge el enfoque de derechos humanos como el resultado de "la discusión jurídica y los esfuerzos por traducir las normas internacionales de derechos humanos en acciones de política institucional y de control social"' (González, 2004: 58).

Este enfoque ha sido desarrollado principalmente por la ONU asegurando que es el camino o medio para hacer posible un desarrollo humano sostenible, logrando empoderar a la ciudadanía y que puedan ejercer sus derechos de la forma adecuada. Para lograr materializar el enfoque de derechos humanos y hacerlo efectivo en la práctica se debe aplicar de forma obligatoria en las políticas públicas y en decisiones judiciales, es decir, que las acciones de los funcionarios del Estado deben estar transversalizadas por los estándares de los derechos humanos, y los derechos deben ser el eje central para lograr su consolidación, sobre todo en aquellas poblaciones vulneradas a través de los años, este enfoque realiza un mayor reconocimiento de los derechos humanos y convenios o tratados internacionales y de las necesidades específicas de la ciudadanía.

El enfoque de derechos humanos es novedoso, pues tiene como objetivo trasladar al ámbito práctico el cumplimiento de los derechos humanos que se encuentran en tratados y convenios internacionales para lograr su verdadera realización, además prioriza los grupos vulnerables, intentando aplicar políticas públicas diferenciadas, por encontrarse en un riesgo mayor, eliminando las barreras de desigualdad que aún subsisten.

El enfoque de derechos humanos según el Alto Comisionado para los derechos humanos se fundamenta en dos aspectos básicos, "el primero, de naturaleza intrínseca, considerando que es lo correcto desde una perspectiva ética y moral y el segundo, de índole instrumental afirmando que reivindica derechos y conduce a mejores resultados" (OACDH, 2006;16). Además, establece las siguientes obligaciones estatales: 1. Primero de respetar, esto quiere 
decir que el Estado no incida o interfiera en la realización plena de los derechos. 2. De proteger, es decir que el Estado realice las acciones pertinentes para evitar la vulneración de los derechos y, por último; cumplir esta exige que el Estado implemente las medidas legislativas, administrativas y judiciales necesarias para promover y garantizar los derechos (OACDH, 2004).

Según la Comisión Interamericana de Derechos Humanos, en su informe "Políticas Públicas con Enfoque de Derechos Humanos" (2018), las políticas públicas son desarrolladas por el aparato institucional y es desde este ámbito donde se deben promocionar los derechos humanos, transformando el diseño e implementación de las políticas públicas de forma transversal, además es deber de los estados cumplir con sus obligaciones en materia de derechos humanos aplicando políticas públicas de forma diligente, por ende las entidades estatales deben realizar las asignaciones presupuestarias priorizando a aquellas poblaciones marginadas, en busca de erradicar la discriminación y pobreza.

Cabe advertir que las políticas públicas pueden definirse como "el reflejo de los ideales y anhelos de la sociedad, expresan los objetivos de bienestar colectivo y permiten entender hacia dónde se quiere orientar el desarrollo y cómo hacerlo, evidenciando lo que se pretende conseguir con la intervención pública y cómo se distribuyen las responsabilidades y recursos entre los actores sociales" (Torres y Santander, 2013: 15). Para que las políticas públicas se desarrollen con enfoque de derechos humanos se deben establecer formas de comunicación adecuadas con la población, establecer proyectos flexibles y adaptables con las necesidades de las personas, identificar situaciones de violencia que requieren de una solución inmediata, facilitar la información y transparencia sobre los diferentes aspectos, con el fin de garantizar que la política pública cumpla su objetivo logrando materializar los derechos humanos.

Así mismo, el enfoque de derechos humanos se fundamenta en los siguientes principios según la CIDH en su informe sobre políticas públicas con enfoque de Derechos Humanos (2018):

1. El principio de igualdad y no discriminación: este hace referencia a garantizar una igualdad material teniendo en cuenta que ciertos grupos se encuentran en desventaja por diferentes factores como la raza, sexo, nacionalidad, y es el Estado mediante la política pública y un trato diferenciado o especifico quien debe eliminar los obstáculos que han impendido la consolidación de sus derechos. Es decir, partir de aceptar las diferencias culturales, sociales y económicas existentes y construir escenarios garantistas para estas poblaciones.

2. Participación social: este principio se basa en la necesidad de modificar el papel tradicional entre el Estado y la sociedad, buscando la participación de los ciudadanos no solo en los mecanismos de participación establecidos en las legislaciones, sino en la construcción de políticas públicas y en la vigilancia o control de las acciones estatales, considerando que una sociedad activa es aquella que participa en la toma de decisiones y en la solución de sus problemáticas.

3. Los mecanismos de reclamo y el acceso a la justicia, este principio es fundamental por obligar al Estado no solo a garantizar los derechos humanos sino a crear los mecanismos idóneos de reclamo ante los jueces o las entidades estatales, cuando estos se vean vulnerados exigiendo una reparación integral y el reconocimiento de los derechos, logrando el acceso a la justicia que por diferentes factores aún en muchos estados no se cumple de la forma indicada. 
4. La protección prioritaria a grupos en situación de discriminación histórica, este principio se relaciona con la necesidad e importancia de diseñar políticas públicas de forma diferenciada, es decir, teniendo en cuenta la discriminación y violencia a la que han estado sometidos ciertos grupos, este planteamiento ha sido desarrollado por la Comisión en varios informes y pronunciamientos indicando la obligación de los estados de adoptar medidas concretas de forma prioritaria con los grupos que han sido vulnerados sistemáticamente.

La Corte IDH, ha emitido varios pronunciamientos y recomendaciones en los que desarrolla el enfoque de derechos humanos por medio de diferentes herramientas como su implementación en políticas públicas y en decisiones judiciales, expresando que (OACDH 2012), en comparación con otros sistemas regionales, no solo propende por la reparación integral en casos concretos, sino por generar mecanismos idóneos de prevención ante las situaciones de discriminación y violencia que se presentan repetitivamente. Así mismo, para la Oficina del Alto Comisionado de las Naciones Unidas para los Derechos Humanos, (2012) el enfoque de derechos humanos facilita el desarrollo de mecanismos pertinentes que enfrentan grandes conflictos sociales, entre ellos la pobreza.

El enfoque de derechos humanos requiere para su aplicación de una política fiscal adecuada que se concentre en suplir las necesidades básicas y en la materialización de los derechos de forma progresiva, es decir, que en las ejecuciones presupuestarias de cada Estado se deben identificar grupos discriminados o vulnerados y asignar el presupuesto de forma diferenciada para lograr mediante políticas públicas correctamente formuladas y diseñadas, la igualdad y cumplimiento en la práctica de todos los derechos.

Por ende, se entiende que la inclusión del enfoque de derechos humanos en la política pública de Colombia, es vital para lograr la consolidación de derechos humanos que en algunas zonas del país han sido vulnerados históricamente, además dado que se está llevando a cabo la implementación del Acuerdo Final de Paz, que busca la reivindicación de grupos sociales y la solución de diferentes problemáticas, el enfoque es un método idóneo para legitimar al Estado en materia de derechos humanos, defender a las poblaciones vulnerables, empoderar a la ciudadanía como sujetos de derechos que tengan la capacidad de participar activamente en la resolución de sus conflictos y en el acceso a la justicia.

\section{EL ENFOQUE DE DERECHOS HUMANOS EN LA IMPLEMENTACIÓN DE UNA POLÍTICA PARA LA PAZ}

Para terminar, el presente acápite quiere mostrar cómo la inclusión del enfoque de derechos humanos permitió la construcción y consolidación de otros enfoques, conocidos como los enfoques diferenciales, los cuales permitirán solucionar los problemas del conflicto atendiendo los interese de la población.

En Colombia se generó conflicto armado, el cual emerge desde los años 60s (Aguilera, 2014) con la creación de varias guerrillas, las cuales se han venido integrando a la sociedad, en el que las FARC-EP (la guerrilla más antigua y más grande) se reincorporó en el año 2016 mediante la firma del Acuerdo Final de Paz, quedando pendiente, hasta el momento, los diálogos con el Ejército de Liberación Nacional (en adelante, ELN). El Acuerdo Final de Paz, puso en evidencia la violencia invisible generada por el Estado. 
Para entender mejor esto, es importante precisar el concepto de violencia que nos ofrece Galtung (1998) desde la teoría sociológica. La violencia según Gatung presenta dos horizontes, la primera es la violencia directa, aquella que es evidente por la secuela de marcas en los cuerpos, y la segunda, es la violencia estructural y cultural la cual se constituye a partir de creencias (violencia cultural) y de instituciones (violencia estructural) (Galtung, 1998).

La violencia directa los colombianos la presenciaban todos los días, con casos como secuestro, desapariciones forzadas por parte de los diferentes actores, ejecuciones extrajudiciales, violencia sexual y formas de esclavitud, etc. La violencia estructural y cultural, la cual es más difícil de ver, se ha concientizado a partir de la firma del acuerdo, así el profesor Aguilera (2014) demostró que el Estado Colombiano, al dejar en desprotección ciertas zonas del país, en donde las políticas públicas no llegaban, conllevó a que consolidara toda una violencia estructural por parte del Estado, puesto que si bien es cierto, en las zonas en las que el Estado no hacia presencia las guerrillas intentaron suplirlo, con la generación de bienes y servicios básicos, esto generó que se precarizaran las condiciones de la población, aumentando la pobreza en el campo, la falta de oportunidades y siendo el campesina estigmatizado por la sociedad civil, al tacharlos de delincuentes.

Demostrado todo este marco complejo de violencia estructural y cultural, en el acuerdo de paz, se introduce la necesidad de igualar las condiciones de las personas en el campo, creando una reforma agraria y diseñando políticas públicas para la generación de desarrollo y seguridad social en el campo. El documento que tiene el lenguaje de la política pública fue traducido al lenguaje jurídico por medio de lo que se conoció como el procedimiento legislativo especial para la paz, con el fin de ser legitimado por fundamentos constitucionales. Así las cosas, el Estado ha generado un sinfín de acciones con el fin de dar cumplimiento efectivo al desarrollo del Acuerdo Final de Paz. Sin embargo, en la práctica este se ha visto entorpecido por actitudes debido al cambio de gobierno y por una radicalización de la violencia estatal en las comunidades campesinas e indígenas que han sido atacados por parte de la fuerza pública ${ }^{8}$ y por parte de fuerzas emergentes del paramilitarismo.

El Acuerdo Final de Paz cuenta con seis puntos específicamente, el primer punto busca establecer un pacto con el campo, y tiene como propósito principal generar equidad en los territorios donde más se ha consolidado la violencia, que permita el desarrollo y progreso en estas zonas. El segundo punto, trata sobre la necesidad de introducir cambios en la forma de hacer política y permitir la inclusión de más actores en el desarrollo de esta. El tercer punto, habla sobre la reincorporación de los miembros de las FARC, el cuarto punto, trata un asunto muy importante para los campesinos, que tiene que ver con la sustitución de los cultivos ilícitos, el quinto punto, es sobre los derechos de las víctimas y todo el esquema de verdad, justicia, reparación y no repetición y el último punto, trata sobre las comunidades étnicas.

En la construcción de los puntos se establecieron concretamente tres enfoques principalmente, estos enfoques son: el enfoque de género, el enfoque étnico y el enfoque territorial $^{9}$. Estos enfoques buscan desarrollar la política pública y la toma de decisión judicial con base en elementos diferenciales que implican los tres enfoques, además de lo anterior, en

\footnotetext{
${ }^{8}$ El más reciente escándalo en dónde el Estado Colombiano se ve envuelto en la violación de derechos humanos, fue el bombardeo del 29 de agosto de 2019, en el que la fuerza pública realizó un ataque a un campamento de los disidentes de la guerrilla de las FARC-EP, en donde se encontraban recluidos ilegalmente 18 niños, y que murieron debido al ataque del Ejército Nacional de Colombia.

${ }^{9}$ Es necesario tener claro que un concepto es los enfoques y otro concepto, son las personas de especial protección. Los dos son conceptos que intentan proteger a las personas, pero tienen elementos distintos. Estudios de la Paz y el Conflicto, Revista Latinoamericana, IUDPAS-UNAH, Número Especial 1, 2019, pp. 27-42. DOI: 10.5377/rlpc.v0i0.9501
} 
la lectura del documento, se encuentran varias referencias al enfoque de derechos humanos, lo cual, también se incluye como obligación para la implementación del Acuerdo. Tal como se muestra en la siguiente tabla (tabla 1), estas referencias son:

Tabla 1. Enfoque de derechos en el Acuerdo Final de Paz

\begin{tabular}{|l|l|}
\hline \multicolumn{1}{|c|}{ Punto } & \multicolumn{1}{c|}{ Referencia } \\
\hline $\begin{array}{l}\text { Sustitución de } \\
\text { cultivos ilícitos }\end{array}$ & $\begin{array}{l}\text { "Enfoque de derechos humanos: las acciones encaminadas a la prevención y } \\
\text { superación del consumo, así como a la mitigación de riesgos y daños, deben } \\
\text { estar enmarcadas en el respeto y goce efectivo de los derechos de las personas } \\
\text { que implica, entre otros, la no estigmatización o discriminación del } \\
\text { consumidor y su no persecución penal en razón del consumo" (Acuerdo Final } \\
\text { de Paz, punto 4). }\end{array}$ \\
\hline $\begin{array}{l}\text { Punto 5. Sobre la } \\
\text { implementación } \\
\text { del sistema de } \\
\text { verdad, justicia, } \\
\text { reparación y no } \\
\text { repetición }\end{array}$ & $\begin{array}{l}\text { "Enfoque de derechos: Todos los acuerdos a los que lleguemos sobre los } \\
\text { contribuir a la proteción y la garantía del goce efectivo de los derechos de } \\
\text { todos y todas. Los derechos humanos son inherentes a todos los seres humanos } \\
\text { por igual, lo que significa que les pertenecen por el hecho de serlo, y en } \\
\text { consecuencia su reconocimiento no es una concesión, son universales, } \\
\text { indivisibles e interdependientes y deben ser considerados en forma global y de } \\
\text { manera justa y equitativa. En consecuencia, el Estado tiene el deber de } \\
\text { promover y proteger todos los derechos y las libertades fundamentales, y todos } \\
\text { los ciudadanos el deber de no violar los derechos humanos de sus } \\
\text { conciudadanos. Atendiendo los principios de universalidad, igualdad y } \\
\text { progresividad y para efectos de resarcimiento, se tendrán en cuenta las } \\
\text { vulneraciones que en razón del conflicto hubieran tenido los derechos } \\
\text { económicos, sociales y culturales" (Acuerdo Final de Paz, punto 5). }\end{array}$ \\
$\begin{array}{l}\text { "(...) En consecuencia, el Estado tiene el deber de promover y proteger todos } \\
\text { los derechos y las libertades fundamentales, sin discriminación alguna, } \\
\text { respetando el principio pro homine, y todos los ciudadanos el deber de no } \\
\text { violar los derechos humanos de sus conciudadanos, atendiendo los principios } \\
\text { de universalidad, igualdad y progresividad" (Acuerdo Final de Paz, punto 6) }\end{array}$ \\
\hline $\begin{array}{l}\text { Punto 6. } \\
\text { Principios } \\
\text { implementación } \\
\text { del Acuerdo } \\
\text { Final de Paz }\end{array}$
\end{tabular}

A partir de lo anterior, sostenemos que, el enfoque de derechos humanos permite la inclusión de los demás enfoques, esto es que el enfoque de los derechos humanos es trasversal a toda la política pública y que en el desarrollo de esta se deben considerar los otros enfoques dependiendo de la población con la que se trabaja o a la que se quiere acercar a través de política pública y de toma de decisión judicial. Los enfoques diferenciales permitirán la construcción del diálogo entre el Estado y las comunidades a partir de las conversaciones entre sociedad civil y Estado, que garantice la solución de los problemas, a partir de los intereses específicos de las comunidades. En conclusión, podemos pensar que el enfoque de los derechos humanos generó la incorporación de los instrumentos nacionales e internacionales sobre derechos humanos, y los enfoques diferenciales específicos, permitirán comprender los problemas desde la diferencia y los intereses concretos de comunidades que han sido vulnerados a lo largo de la historia. A saber (figura 1): 
Figura 1. Construcción de los enfoques

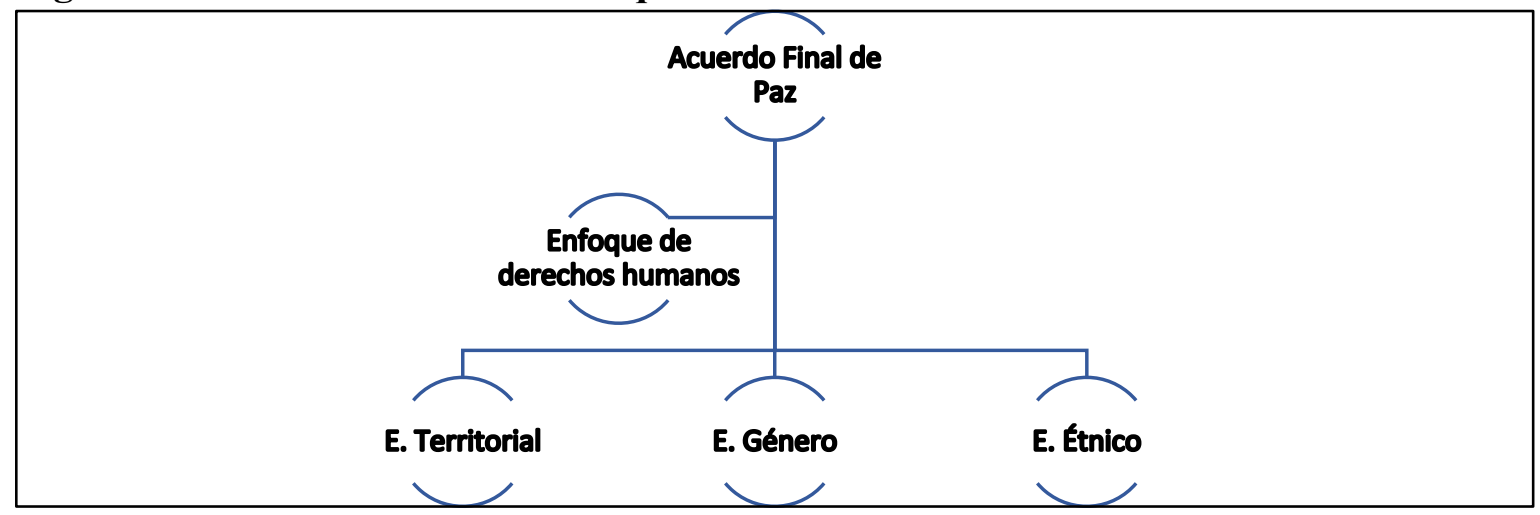

Ahora bien, respecto de cada uno de los enfoques, el enfoque territorial permitirá la construcción de la política pública a partir de los intereses específicos del territorio, especialmente de aquellos territorios que han estado abandonados por parte del Estado. En el que requieren de la atención urgente por parte de las instituciones públicas. Este enfoque conllevará a la formación de un puente entre lo que la comunidad necesita y la política pública.

Por otro lado, el enfoque de género, permitirá mostrar cómo cierta población se ha encontrado en condiciones de discriminación y de vulneración que surgen de creencias y de prácticas violentas naturalizadas. El Estado tendrá que responder a la problemática con base en estos matices de violencia indirecta o estructural (Galtung, 1998), que implica la superación de esta, para materializar el derecho a la igualdad de las diferentes personas, especialmente de las mujeres y de las personas con diversidad sexual no tradicionales, quienes sufren de una violencia específica, como lo demostró el informe "La guerra inscrita en el cuerpo", elaborado por el Centro Nacional de Memoria Histórica.

Por último, el enfoque étnico, se sustenta en las teorías contemporáneas del multiculturalismo, el cual busca responder a las concepciones y formas de vida diferentes, lo cual requiere de una aplicación de los derechos humanos, dependiendo de las ontologías de ser diferenciadas, que permitirán la materialización del bienestar en la comunidad. El enfoque étnico hace referencia a tres comunidades específicamente, las comunidades indígenas, el pueblo Rrom y los negros, afrocolombianos, palenqueros y raizales.

El enfoque de los derechos humanos cerrará la concepción de los demás enfoques diferenciales, porque la base de los enfoques se encuentra sustentado en la materialización efectiva del corpus iuris internacional de los derechos humanos, es decir, la aplicación de valores universales construidos para generar bienestar en la sociedad, y los enfoques diferenciales específicos, permitirán que los derechos universales no se construyan desde arriba hacia abajo, es decir, desde lo que el Estado considera que es bueno o malo, sino que los enfoques diferenciales, como el de género, territorio y étnico, permitirán la materialización de los derechos a partir de los intereses específicos de la comunidad, de las mujeres, de los indígenas, etc.

En términos teóricos podríamos decir, que los enfoques diferenciales, tales como el enfoque de género, territorial y étnico son el medio que supera la disputa entre el universalismo y el relativismo, y que permite un diálogo entre la comunidad y la 
materialización de valores universalmente reconocidos. Es decir, el derecho a través de los enfoques se convierte en el puente entre lo subjetivo y lo universal.

En términos prácticos el derecho se convierte entonces en aquello que parece insuperable en la filosofía, esto es la disputa entre lo universal y lo particular, para no instalarse en ninguno de los extremos sino negociar entre los intereses específicos de la comunidad y la materialización de lo que los Estados consideran como valores universales.

\section{CONCLUSIONES}

Los derechos humanos son instrumentos vigentes, que deben estar presentes en cada una de las leyes, actos administrativos, reglamentos y demás pronunciamientos y acciones tanto del Estado como de particulares, ya que son una obligación constitucional y supraconstitucional donde se protegen a los seres humanos en su singularidad, teniendo en cuanta sus diferencias y características específicas. Son mandatos de los cuales no se puede prescindir porque buscan establecer las condiciones básicas de vida y dignidad humana para todas las personas, y su violación acarrea graves perjuicios que deben ser subsanados y reparados.

El enfoque de derechos humanos es un mecanismo útil, novedoso y pertinente para reivindicar los derechos humanos de aquellos grupos que han sufrido históricamente situaciones de violencia, además consolida la aplicación de convenios y tratados internacionales en la práctica por medio de su inclusión en las políticas públicas y decisiones judiciales, es decir, que soluciona problemáticas y conflictos sociales de forma diferenciada, con el fin de garantizar no solo los derechos sino un nivel de vida digno para todas las personas.

El enfoque de los derechos humanos al ser el enfoque más tradicional generó la obligación de desarrollar dentro de la política pública la introducción de los instrumentos internacionales, con el fin de consolidar y hacer efectivo cada uno de los derechos en la práctica. Además, permitió dentro de la construcción de una política para la paz, como lo fue el Acuerdo Final de Paz, la consolidación de otros enfoques, estos son: enfoque étnico, enfoque de género y enfoque territorial, los cuales permitirán la generación de políticas públicas y la toma de decisión judicial con base en los intereses específicos de la comunidad. Los enfoques en consecuencia se consolidan como un puente entre el desarrollo universal de unos valores, a saber: los derechos humanos y su concreción dependiendo de los valores y la cultura, garantizando un diálogo entre la sociedad civil y el Estado.

\section{REFERENCIAS}

Aguilera P. (2014). Contrapoder y justicia guerrillera. Fragmentación Política u orden insurgente en Colombia (1952-2003). Bogotá: Universidad Nacional de Colombia.

Atienza, M. (2019). Interpretación Constitucional. Bogotá: Universidad Libre.

Alexy, R. (2008). Principio de Proporcionalidad y la interpretación constitucional. Quito: V\&M Gráficas.

Arendt, H. (2014). La condición humana. Barcelona. Paidós.

Bobbio, N. (1957). Voce Principi generali di diritto. Roma: Novissimo Digesto Italiano. Centro Nacional de Memoria Histórica. (2017). La guerra inscrita en el cuerpo. Informe. 
CIDH (Comisión Interamericana de Derechos Humanos) (2018). Políticas públicas con enfoque de Derechos Humanos. CIDH (Disponible online: http://www.oas.org/es/cidh/informes/pdfs/PoliticasPublicasDDHH.pdf)

Ferrajoli, L. (2012). Un debate sobre el Constitucionales. Buenos Aires: Marcial Pons.

Ferrajoli, L. (2016). Derechos fundamentales. Democracia Constitucional y garantismo". Bogotá: RZ.

Galtung, J. (1998). Tras la violencia, 3R: Reconstrucción, reconciliación, resolución. Afrontando los efectos visibles de la guerra y la violencia. Bilbao: Gernika Gogoratuz.

González, P. (2004). Los derechos humanos como centro de las políticas públicas: apuntes teóricos y prácticos. Caracas: Provea, Mimeo.

Hart, H. (2004). El concepto de derecho. Segunda edición. Buenos Aires: Abeledo-Perrot.

OACDH (Oficina del Alto Comisionado de las Naciones Unidas para los Derechos Humanos) (2004). Los derechos humanos y la reducción de la pobreza: un marco conceptual. Ginebra y Nueva York: Oficina del Alto Comisionado de las Naciones Unidas para los Derechos Humanos

OACDH (2006). Preguntas frecuentes sobre el enfoque de derechos humanos en la cooperación para el desarrollo. Ginebra: Oficina del Alto Comisionado de las Naciones Unidas para los Derechos Humanos.

ONU (Organización de las Naciones Unidas) (1948). Declaración Universal de los Derechos Humanos, aprobada por la Resolución 217A, de 10 de diciembre de 1948. Ginebra: ONU (Disponible en https://www.un.org/es/sections/issues-depth/human-rights/index.html).

Niño, N. (2018). Discusiones sobre la implementación del acuerdo final de paz: Una mirada en perspectiva de la ciencia social. Bogotá: Universidad Libre.

Niño, N. (2019). "Perspectiva y enfoque de género: herramienta para la toma de decisión

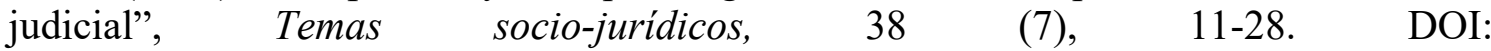
https://doi.org/10.29375/01208578.374.

Torres, J., Santander, J. (2013). Introducción a las políticas públicas: conceptos y herramientas desde la relación entre Estado y ciudadanía. Bogotá: IEMP Ediciones.

\section{Normativa y jurisprudencia}

Constitución Política de Colombia de 1991, Bogotá, Gaceta Constitucional nº 114 del jueves 4 de julio de 1991.

Decreto Ley 121 de 2017, por medio del cual se establecen instrumentos jurídicos para facilitar y asegurar la implementación y el desarrollo normativo del Acuerdo Final para la Terminación del Conflicto y la Construcción de una Paz Estable y Duradera (Disponible en:

http://es.presidencia.gov.co/normativa/normativa/DECRETO\%20121\%20DEL\%2026\% 20ENERO\%20DE\%202017.pdf).

Convención de Delegados de Virginia, Declaración de Derechos de Virginia, 1776. Recuperado de: file://C:/Users/Home/Downloads/DialnetLaDeclaracionDeDerechosDeVirginia12DeJunioDe1776-2062239.pdf

Asamblea Nacional de Francia, Declaración de los Derechos del Hombre y del Ciudadano, $1789 . \quad$ Recuperado de: https://www.conseilonstitutionnel.fr/sites/default/files/as/root/bank_mm/espagnol/es_ddhc.pdf

Congreso Constituyente de Querétaro, Constitución Política del estado de Querétaro, 1917. 
Recuperado

de: https://armonizacion.cndh.org.mx/Content/Files/sec03 A/constitucion/Constitucion_Qro .pdf

Conferencia Especializada Interamericana sobre Derechos Humanos, Convención Americana sobre Derechos Humanos, 1969. Recuperado de: https://www.oas.org/dil/esp/tratados b32 convencion_americana_sobre derechos hum anos.htm

Organización de los Estados Americanos, Convención Interamericana Para Prevenir Y Sancionar la $\quad 1985 . \quad$ Tortura, Recuperado de: https://www.oas.org/juridico/spanish/tratados/a-51.html

Organización de las Naciones Unidas, Convenios de Ginebra, 1949. Recuperado de: https://www.oas.org/juridico/spanish/tratados/a-51.html

Organización de las Naciones Unidas, Convenios de Ginebra y el Protocolo Adicional I, 1977. Recuperado de: https:/www.icrc.org/es/document/protocolo-i-adicionalconvenios-ginebra-1949-proteccion-victimas-conflictos-armados-internacionales-1977

Sentencia C-067/03 de la Corte Constitucional de Colombia, de 4 de febrero de 2003 (M.P.: M. G. Monroy).

Sentencia C-579/13 de la Corte Constitucional de Colombia, de 28 de agosto de 2013 (M.P.: J. I. Pretelt).

Sentencia de 15 septiembre de 2005, de la Corte Interamericana de Derechos Humanos (caso de La Masacre de Mapiripan Vs. Colombia) (Disponible en: http://www.corteidh.or.cr/docs/casos/articulos/seriec_134_esp.pdf).

Congreso de Estados Unidos, Bill of Rights, 1789. Recuperado de: https://www.archives.gov/founding-docs/bill-of-rights-transcript

\section{REFERENCIA DE LAS AUTORAS}

Natali Niño Patiño, Filósofa y abogada. Especialista en Filosofía del Derecho y Teoría Jurídica, magíster en Filosofía del Derecho y Teoría Jurídica. Estudiante del Doctorado en Derecho de la Universidad de Buenos Aires. Docente investigadora del Observatorio de Paz de la Universidad Libre. Docente en pregrado de Historia de la Filosofía y Lógica Jurídica. miembro del Grupo de Investigación de Estudios Constitucionales y de la Paz, docente de investigación del Instituto de Posgrados de la Universidad Libre.

natali.ninop@unilibre.edu.co.

Laura Milena Aldana González, estudiante de quinto año de Derecho de la Universidad Libre. Miembro del Observatorio de Paz de la Universidad Libre. Monitora del área de sociales. lauram-aldanag@,unilibre.edu.co.

María Camila Zuluaga, estudiante de tercer año de Derecho de la Universidad Libre. Miembro del Observatorio de Paz de la Universidad Libre. mariac-zuluagag@unilibre.edu.co.

Recibido: 10-11-2019

Aceptado: 04-02-2020

(c) (i) Licencia Creative Commons Reconocimiento 4.0 (CC BY 4.0).

Estudios de la Paz y el Conflicto, Revista Latinoamericana, IUDPAS-UNAH, Número Especial 1, 2019, pp. 27-42. DOI: 10.5377/rlpc.v0i0.9501 